\title{
Efficient Garbage Disposal Management in Metropolitan Cities Using VANETs
}

\author{
Narendra Kumar G., Chandrika Swamy, and K. N. Nagadarshini
}

\begin{abstract}
Rapid increase in population, has led to improper waste management in metropolitan cities resulting in increased pests and spreading of diseases. An efficient method to dispose this waste has been designed with Wireless Sensor Networks (WSN) using VANETs. IEEE 802.11p has been adopted and multicast routing has been proposed to be implemented in Garbage Collecting Vehicle's (GCV) On Board Units (OBU) for effective communication. Road Side Units (RSU) and sensors have been made use of in the response system. Filling up of multiple bins at the same time and usage of reserve GCVs has been considered. The prototype VANET based efficient garbage disposal system is induced in a metropolitan city environment and has been simulated in NS2and the results are encouraging for implementation.
\end{abstract}

Index Terms - Garbage collecting vehicle (GCV), road side unit (RSU), vehicular Ad-Hoc network (VANET), wireless access in vehicular environment (WAVE).

\section{INTRODUCTION}

Waste is defined as any material that is not useful and represents no economic value to its owner, the waste generator. Depending on the physical state of waste, they are categorized as solid, liquid and gaseous.

Waste Management involves planning, financing, construction and operation of facilities for the collection, transportation, recycling and final disposition of the waste. Every five years the waste generated is rising by 1 million ton, Fig. 1. In case it is not disposed within a stipulated time, it tends to create serious health hazards and reflects negatively on the infrastructure. The existing garbage disposal system, where it is collected from the streets, houses and other establishments once a day, is not able to effectively manage the waste generated, resulting in spill over on streets.

Bangalore metropolitan city municipal council deployed concrete dustbins at every street corner to collect the garbage, engaged its labourers and vehicles to clear the trash. The municipal efforts did not pay any dividend and hence it had to eliminate the bins since residents would litter garbage around the bin once the bins were full. Consequently concrete dustbins were replaced by the door-to-door garbage collection system, which was also ineffective in its implementation.

The Indian National Capital Territory, Delhi is rapidly growing and so is its corresponding waste production. $85 \%$ of the city does not have a formal door-to-door trash pick-up system. The Municipal waste, which is not always properly

Manuscript received June 3, 2013; revised July 22, 2013.

The authors are with Department of Electronics and Communication Engineering, UniversityVisvesvaraya College of Engineering, Bangalore University, India (gnarenk@yahoo.com, chandrikaswamy91@gmail.com, nagadarshini1991@gmail.com). segregated at the source, often ends up as mixed waste in the already overflowing landfills.

Solid WasteProjected (at 300 grms per capita per day)

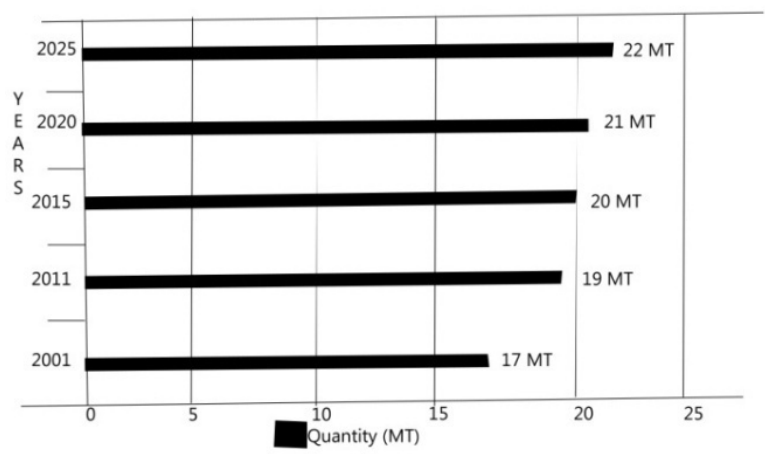

Fig. 1. Statistics of solid waste projected (at 300 gm per capita per day)

Along with the recent economic boom in China, the quantity of solid waste generated is increasing multi-fold, posing a huge challenge for environmental management thereby exacerbating the problem of solid waste management. The need for intelligent waste management has led to the concept of the hierarchy of waste management that places the various means for dealing with Municipal Solid Waste (MSW) in order of environmental preferences. Waste To Energy (WTE) is proving to be an unabated technology in China. Of the estimated one billion ton (907 million tonnes)of global post-recycling MSW, close to 200 million tons (181million tonnes) are processed in WTE plants that recover the energy content of waste in the form of electricity or heat, [1].

In Singapore, the door-to-door collection of garbage has been outsourced to four Public Waste Collectors (PWC). Thegovernment collects a uniform fee from the residents for the same. In case a member of the public observes illegal dumping of any kind, he/she can contact a call centre and provide the respective information to the concerned department.

Improper waste management and disposal causes serious impact on health, leading to the spread of infectious diseases and problems to the surrounding environment. Organic domestic wastes pose a serious threat, since they ferment, creating favourable conditions for the survival and growth of microbial pathogens. Exposure to hazardous waste affects human health, children being more vulnerable to these pollutants. Uncollected solid waste obstructs storm water runoff, resulting in the formation of stagnant water bodies that becomes breeding grounds of diseases. Waste dumped near a water source causes contamination of the water body and the ground water source. Direct dumping of untreated waste in rivers, seas and lakes results in the accumulation of 
toxic substances in the food chain through the plants and animals. Waste treatment and disposal sites create health hazards for the neighbourhood causing air pollution and attracting all types of insects and rodents that spread disease.

In this paper, a model has been proposed in which the collection of garbage is made real time. A network is established using wireless sensor nodes with each dustbin attached to a sensor circuitry, which continuously senses the status of the dustbin and sends a signal to the nearest Road Side Unit (RSU) when it is full. The RSU further transmits the same signal to other RSUs until it reaches the Garbage Collecting Vehicle (GCV) which arrives at the place where the dustbin is located and collects the garbage. Each block of the proposed WSN and the transmitter is explained in brief and the process is elaborated with a flowchart.

\section{EXISTING WORK}

In existing models, real time monitoring of status of bins, estimation of amount of waste in and around them, surveillance for monitoring the movement of vehicles, optimization of routes and reallocation of bins according to the estimated waste, availability of Management Information System (MIS) reports for effective planning of resource schedule and providing transparency in civic administration are dealt with.

Each container is equipped with a Radio Frequency Identification (RFID) label having a unique identification code, the Electronic Product Code (EPC). Low frequency passive tags are proposed as they offer long term low cost solutions and are operational in extreme conditions resistant to environmental hazards. As the container gets loaded onto the truck, the RFID reader reads the serial number of the tag on the container, and at the same time, the Global Positioning System (GPS) receiver on the truck calculates its location using satellite data. The serial number of the tag, location, date and time are transmitted real time via the Global System for Mobile (GSM) network to the communication gateway of the control server. After data processing, it is transferred to the Global Information System (GIS) terminal. The real time information can be shared with clients via a web based solution, [2].

\section{A. Vehicular Ad-Hoc Networks (VANETs)}

VANETs are a class of Mobile Ad-Hoc Networks (MANETs). They can provide communication between the vehicles (nodes) i.e. Vehicle-to-Vehicle (V2V) communication and/or communication between vehicles and the roadside infrastructure, Vehicle-to-Infrastructure (V2I) communication. The vehicles and the associated infrastructure (all nodes) are equipped to gather data, process it to determine present traffic conditions and disseminate it over longer distances and provide other traffic related services related to toll ticketing, monitoring, collision warning, road signal alarms and so on. VANETs help provide safety and comfort for passengers through intelligent use of networking. Recent research efforts have placed a strong emphasis on novel VANET design architectures and implementations. Emphasis has been laid on areas like broadcasting, routing, security and Quality of Service (QoS), [3].

\section{B. Wireless Sensor Networks (WSN)}

A WSN consists of spatially distributed autonomous sensors to monitor physical or environmental conditions, such as light, temperature, sound, vibration, pressure, humidity, motion or pollutants and to cooperatively pass their data through the network to a main location. The WSN is built of nodes from a few to several hundreds or even thousands, where each node is connected to one or several sensors. Each such sensor network node typically has several parts: a radio transceiver with an internal antenna or connection to an external antenna, a microcontroller, an electronic circuit for interfacing with the sensors and an energy source, usually a battery or an embedded form of energy harvesting, [4], [5].

\section{WIRELESS ACCESS IN VEHICULAR ENVIRONMENT (WAVE)}

Vehicular traffic scenarios have greater challenges than fixed wireless networks, caused by varying driving speeds, traffic patterns, and driving environments. To address challenging requirements of IEEE MAC operations for vehicular communication scenario, IEEE802.11p Wireless Access in Vehicular Environments (WAVE) was introduced. $802.11 \mathrm{p}$ is an IEEE standard that supports Intelligent Transportation Systems, Vehicle to Vehicle communication (V2V) and Vehicle to Infrastructure communications (V2I) that are being developed, namely the Dedicated Short Range Communications (DSRC) operating in $5.9 \mathrm{GHz}$ band. WAVE has become a standard that can be universally adopted across the world compared to the regional standards of DSRC. At present DSRC based on the Wi-Fi standard is widely used in VANETs as it connects infrastructure to vehicles and also vehicles-to-vehicles using two way short range radio which is of lower costs compared to other wireless standards available. DSRC/WAVE systems fill a niche in the wireless infrastructure by facilitating low latency, geographically local, high data rate, and high mobility communications.

WAVE defines two types of devices: Road Side Unit (RSU), and On Board Unit (OBU) which are essentially stationary and mobile devices respectively. RSUs and OBUs can be either a provider or a user of services and can switch between such modes, [6], [7].

\section{Proposed Garbage Disposal Management Using VANETS}

\section{A. Assumptions}

C. Existence of a well-connected network of segregated dustbins -dry, wet and plastic, placed at strategic locations and presence of wireless connection between RSUs and sensors.

D. Existence of Road Side Units (RSU) at suitable locations in order to transmit signals.

E. Presence of a centrally located Base Station (BS) that monitors the entire garbage collection and disposal module.

F. Existence of a periodically updated database of status of all the dustbins and current location of Garbage Collecting Vehicle (GCV). 


\section{B. Requirements for the Proposed Model}

\section{1) Dustbins}

Dustbins are the primary infrastructure required. Separate dustbins are allocated for dry and wet waste respectively, each of which is equipped with suitable low power sensor circuitry to detect the fullness and heaviness, and transmitters to send a message to the nearest GCV when it is full.

\section{2) Sensor circuitry}

\section{LASER Diode}

A LASER diode is a $\mathrm{p}-\mathrm{n}$ junction diode which produces a narrow beam of light that is intense, focused and coherent. In a LASER diode a mirrored resonant chamber is used to reinforce the light waves so that the light emitted by the device is at a single frequency and of the same phase. The two ends of the structure need to be optically flat and parallel with one end mirrored and one partially reflective. The length of the junction must be precisely related to the wavelength of the light to be emitted, Fig. 2.

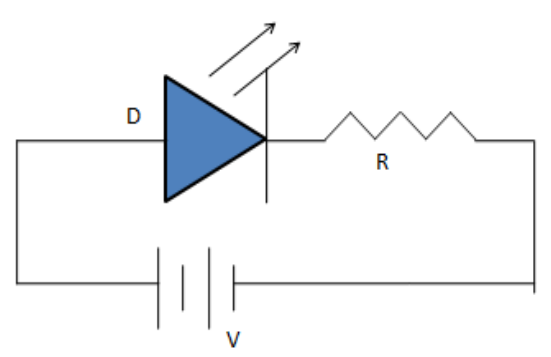

Fig. 2. LASER Diode

\section{Photo Detector Diode}

A photo detector is a device that converts light signals into electrical signals, which can be amplified and processed. During absence of light, the reverse bias draws current carrying electrons and holes out of the p-n junction region, creating a depleted region, which stops current from passing through the diode, Fig. 3.

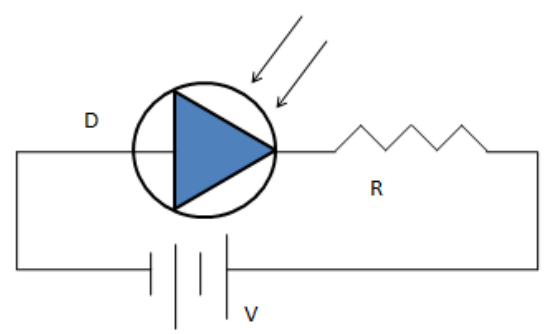

Fig. 3. Photo detector Diode

On incidence of light on the detector, photons with the proper energy (wavelength) can create electron-hole pair. The bias voltage causes these current carriers to drift quickly away from the junction region, so the current flow is proportional to the intensity of light incident on the detector. The wavelengths at which the detector responds to light depend on the detector's material composition, [8].

\section{1) $\mathrm{RC} 11 \mathrm{XXHP}-\mathrm{RC} 232$}

The RC11XXHP-RC232 RF Transceiver Modules are compact surface-mounted high performance modules for FSK operation with embedded protocol. The modules are completely shielded and pre-certified for operation under the European and Indian radio regulations. Its features include:
High Power, long range (3-5 km Line-Of-Sight); Completely Shielded compact 7 × 25.4 × $3.3 \mathrm{~mm}$ module for Surface Mount Device (SMD) mounting; No external components; 2.7-3.3 V supply voltage, ultra-low power modes, Fig. 4.

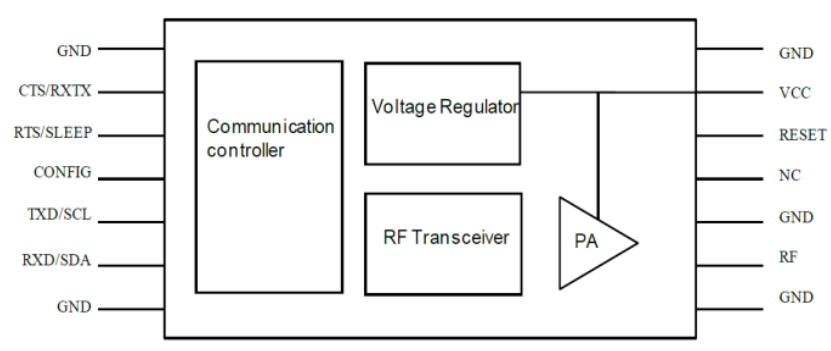

Fig. 4. RF transceiver block diagram

\section{2) Road Side Units (RSU)}

RSU acts similar to a wireless LAN access point and can provide communications with infrastructure. Also, if required, RSU must be able to allocate channels to OBUs. There is a third type of communicating nodes called Public Safety OBU (PSOBU) which is a vehicle with capabilities of providing services normally offered by RSU. These units are mainly utilized in police cars, fire trucks, and ambulances in emergency situations. In the current model, each RSU, being an access point receives the information from the dustbins/other RSUs in the vicinity of its radio range.

\section{3) Garbage Collecting Vehicle ( $\mathrm{GCV}$ )}

A fleet of GCV is deployed in various locations depending on the population density of the area. Each of these is equipped with a RC11XXHP-RC232 transceiver module which communicates with the nearest RSU and subsequently the BS.

The components of the proposed system is depicted, Fig. 5

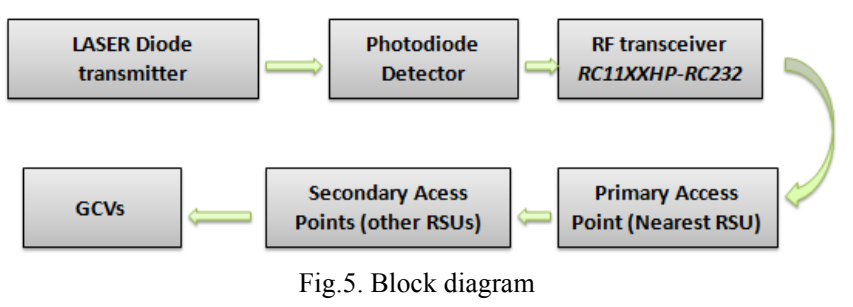

\section{IMPLEMENTATION}

Each street is provided with a dustbin (separate for dry and wet waste) and residents are instructed to dump the garbage in these bins only. Each of such dustbins is equipped with a light sensor circuitry at three fourth distances from base of dustbin, so that by the time the bin is completely full, the GCV arrives and there is no scope for spillage.

The LASER diode, powered by a low power replaceable battery, transmits light to the photodiode, placed at its line of sight directly opposite to the transmitter. The circuit is designed not to send any signals as long as the detector receives light continuously from the transmitter.

The dustbin being full, the light from the LASER diode is blocked by the garbage being filled into it. The photodiode upon not receiving light for a predefined amount of time triggers the transmitter RC11XXHP-RC232to send a signal to the nearest RSU, indicating that the dustbin is almost full. 
The neighboring RSUs help in propagation of the signal through the shortest path to the GCV through the network adopting Djikstra's algorithm, thus resolving the shortest path problem. Once the GCV deployed for the concerned location receives the signal from the nearest RSU, it arrives at the location of the dustbin, collects the garbage and replaces it with an empty dustbin. In case a GCV is full, the BS is informed that it is no longer in service as it is going towards the respective dump yard, and hence ignores any other signal received by it. TheBS makes effective allocation of an alternate GCV as a substitute, for a short period of time, Fig. 6.

\section{Simulation AND RESUlts}

Network Simulator-2 (NS-2) a discrete event simulator targeted at networking research provides substantial support for simulation of Transmission Control Protocol (TCP), routing and multicast protocols over wired and wireless (local and satellite) networks. NS-2 was built in $\mathrm{C}++$ and provides a simulation interface through OTcl, an object oriented dialect of $\mathrm{Tcl}$. The user describes a network topology by writing OTcl scripts and then the main NS-2 program simulates that topology with specified parameters. It runs on Linux, FreeBSD, Solaris, Mac OS $\mathrm{X}$ and on Windows using Cygwin.

\section{A. Case Study}

\section{1) Network topology and dustbins filling}

A typical layout of a city is mapped. The blue node represents the BS, maroon nodes represent the RSUs, yellow nodes represent the vehicles allocated to collect dry wastes, green nodes represents the GCVs allocated to collect wet wastes, white nodes represent the empty bins and red nodes represent the filling of the bins. Over a period of time, filling of the bins are depicted by the white nodes changing to red, Fig. 7.

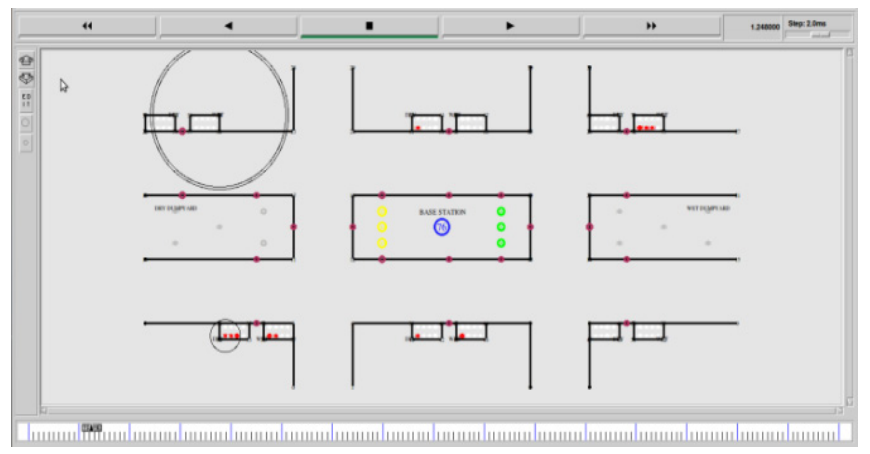

Fig. 7. Network topology

\section{2) RC11XXHP-RC232 Signaling to the nearest $R S U$}

Dustbins 1 dry, 6 wet are $3 / 4^{\text {th }}$ full. The respective transmitters are seen sending a signal to their nearest RSUs. The signal reaches the GCV propagating via intermediate RSUs, Fig. 8.

\section{3) $R C 11 X X H P-R C 232$ Signaling to the nearest $R S U$}

Dustbins 1 dry, 6 wet are $3 / 4^{\text {th }}$ full. The respective transmitters are seen sending a signal to their nearest RSUs. The signal reaches the GCV propagating via intermediate RSUs, Fig. 8.

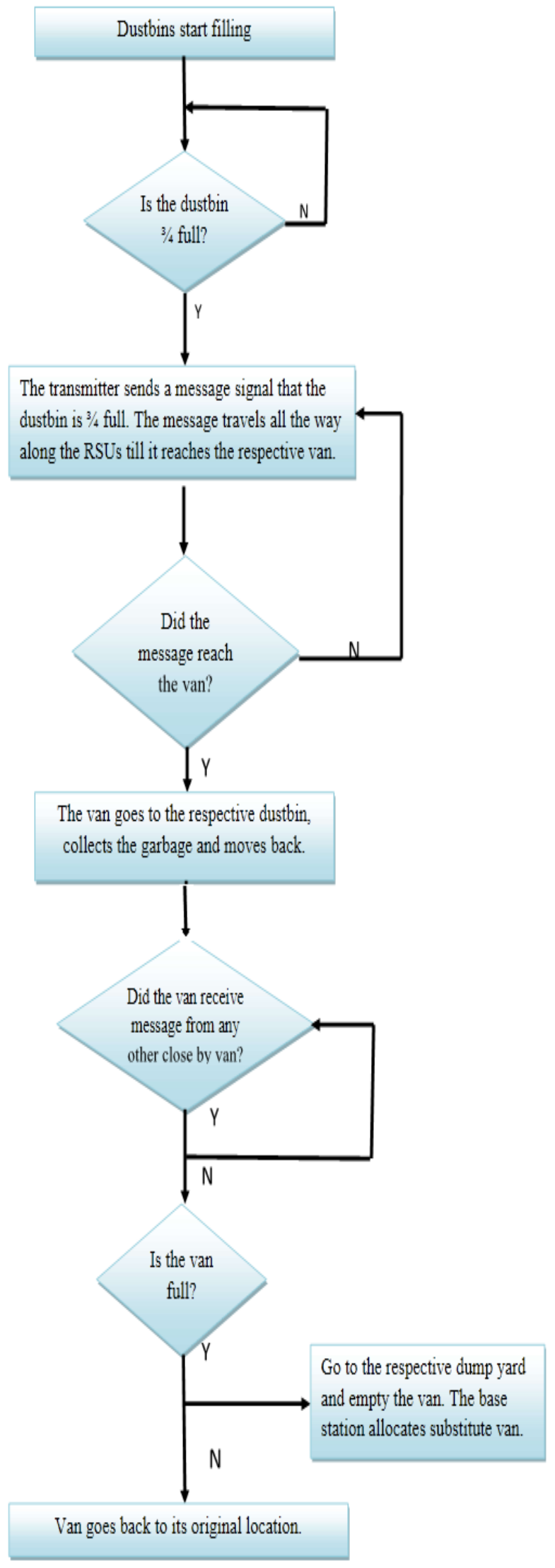

Fig. 6. Flowchart of Implementation

\section{4) GCV being signaled}

The GCV is signaled by its nearest RSU, in response to which it goes to the particular dustbin which is full so as to replace the dustbin, depicted by the red nodes changing to white, Fig. 9. 


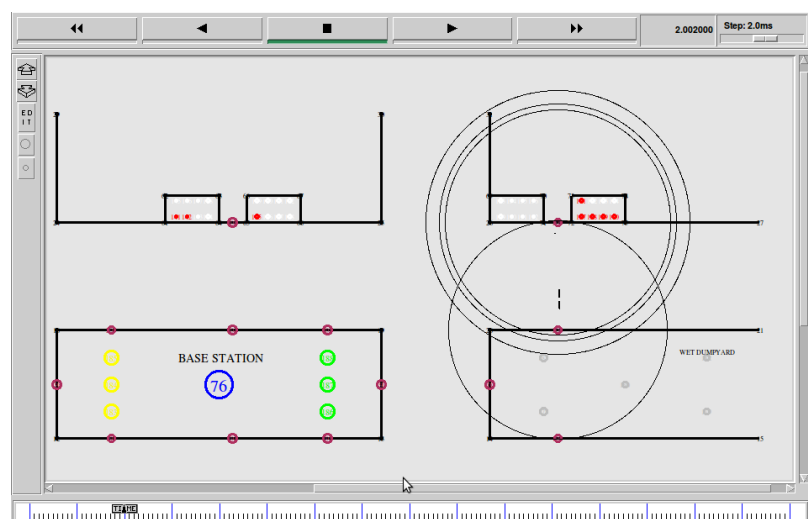

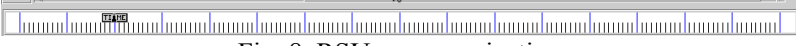
Fig. 8. RSUs communicating

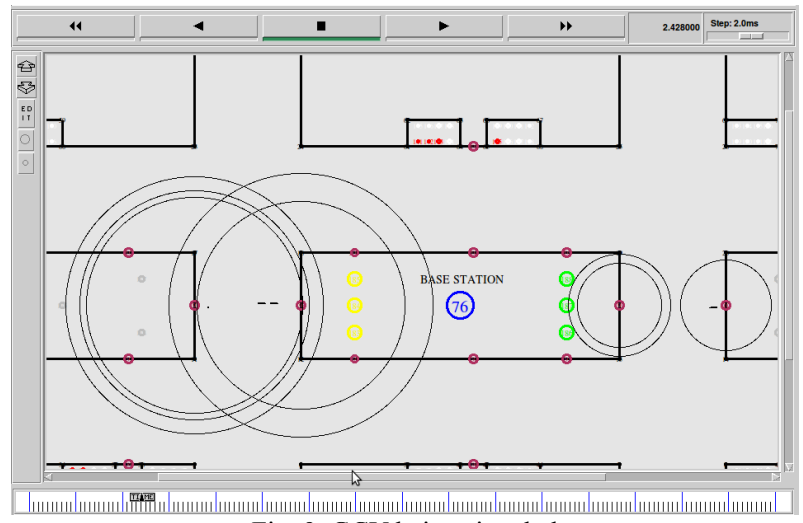

Fig. 9. GCV being signaled

\section{5) GCV returning to the Dump yard}

The black node represents the GCV informing the BS for an alternate as it is in transit to the respective dump yard to empty the trash, Fig.10 and Fig. 11 shows the BS signaling the auxiliary GCV to take over while GCV goes to dump yard.

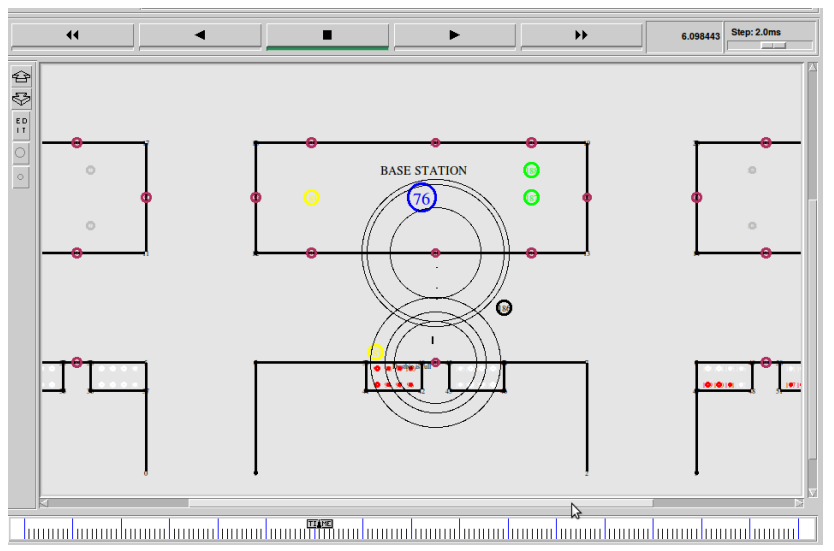

Fig. 10. GCV signaling BS before going to dump yard

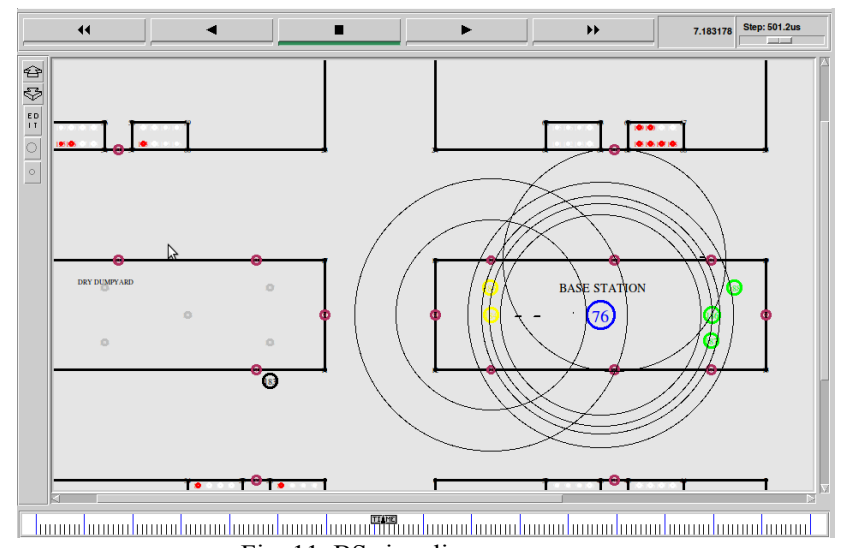

Fig. 11. BS signaling reserve van

\section{CONCLUSION}

This study addresses the problem of clearing the garbage overflowing on roads and proposes a system in which emptying of dustbins is made dynamic by the integration of sensors and infrastructure services. The results of this study illustrate that dynamic routing of GCV compared with static solution is much more efficient and will be much effective when more than one dustbin fills up at the same time. The initial planned route is saved so that when real-time data is received only portion of the planned path may be changed. Real time monitoring with the help of sensors and wireless communication is used as a powerful functionality for planning optimal routes based on available travel time information, indicating that garbage overflow on roads can be prevented by determining the filling up of dustbins at right time. The dustbins are cleared as and when they are filled, thus giving way to a cleaner city, better infrastructure and increased hygiene.

\section{REFERENCES}

[1] Q. F. Huang, Q. Wang, L. Dong, B. D. Xi, and B. Y. Zhou, "The current situation of solid waste management in China", Journal of Material Cycles and Waste Management - JMATER CYCLES WASTE $M A N A G$, vol. 8, no. 1, pp. 63-69, 2006.

[2] S. S. Purohit, "RFID-based solid waste collection,"Recent Advances in Intelligent Computational Systems (RAICS), vol. 22, no.2, IEEE, July 2012.

[3] K. Kolla, R.Rakesha, S.Tejus, and G. Narendra Kumar, "Real time incubator monitoring system using wireless sensor network (2012)," in Proceedings of the 2012 International Conference on Wireless Networks, Las Vegas Nevada, USA, 2012.

[4] M. Gupta, D. Prasad, and R. B. Patel. "FREEDOM: Fault revoking and energy efficient protocol for the deployment of mobile sensor nodes in wireless sensor networks", International Journal of Advanced Engineering Sciences And Technologies, 2010.

[5] A. S. Bhat, B. Raghavendra, and G. N. Kumar, "Enhanced passive RFID based disaster management for coal miners, "International Journal of Future Computer and Communication 2013, vol. 2, no. 5, October 2013, Singapore, 2013.

[6] B.SmithaShekar, C. K.Divyashree, G. George, H. V. Usha Rani, A. Murali, and G. Narendra Kumar, "GPS Based Shortest Path for Ambulances using VANETs," in Proc. International Conference on Wireless Networks (ICWN 2012), vol. 49, Singapore, 2012.

[7] M. Aparajitha, K. Bhanupriya, B. Smitha Shekar, and G. Narendra Kumar, "Performance evaluation of IEEE $802.11 \mathrm{p}$ for vehicular traffic congestion control (2011)", Journal of Information and Communication Technologies, vol. 1, issue 6, November 2011.

[8] L. J. Yin, Q. Chen, S. F. Kou, and J. Qin, "Research on avalanche photodiode based photon imaging system," in Proc. The International Symposium on Photonics and Optoelectronics (SOPO 2009), China, 2009 .

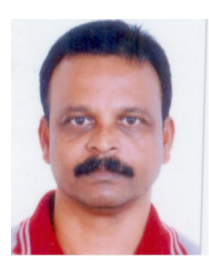

Narendra Kumar G. is a professor in Bangalore University, Bangalore, India. He completed his Master of Engineering Degree in Electrical Communication Engineering, (Computer Science \& Communication) in1987 at IISc, Bangalore, India. Later in 2006 he obtained $\mathrm{PhD}$ in Electrical Engineering (Computer Networks) in 2006 in Bangalore University, Bangalore. His fields of specialization include -Wireless Networks, Multimedia System, Mobile Communication and Disaster Management. 